Revista

\title{
Multi-Ensayos
}

Vol. 5, $\mathrm{N}^{\circ} 10$

ISSN: 2412-3285

https://multiensayos.unan.edu.ni

DOI: https://doi.org/10.5377/multiensayos.v5i10.8873

\section{Metodologías implementadas en el aula de clase, con estudiantes de segundo año de la carrera Administración de empresas}

\section{Methodologies implemented in the classroom, with sophomores of the business administration career}

Donald Ariel Hernández Muñoz

Recibido: 03 de junio de 2019. Aceptado: 30 de junio de 2019

\section{RESUMEN}

Este ensayo fue realizado con el propósito de describir las experiencias vividas en el aula de clase, así como la metodología implementada y dificultades presentadas durante todo el proceso de enseñanza aprendizaje, en la asignatura Estadística l, con estudiantes de la carrera de segundo año de Administración de empresas del turno por encuentro (sabatino). Según lo observado, las metodologías de enseñanza permiten que los estudiantes relacionen la teoría con la práctica, faciliten la compresión de las temáticas abordadas, propicien curiosidad para realizar comprobaciones, investigaciones y aplicar los conocimientos adquiridos en la resolución de problemas.

Palabras claves: Estadística; estrategias; resolución de problemas.

\section{ABSTRACT}

This essay was conducted with the purpose of describing the practices experienced in the classroom, as well as the methodology implemented and difficulties presented throughout the teaching - learning process, in the subject Statistics I, with students from the second-year of Business Administration course (Sabbatine). As noted, teaching methodologies allow students to relate theory to practice, facilitate the understanding of the topics addressed, encourage curiosity to carry out checks, research and apply knowledge acquired in problem solving.

Keywords: Statistics; strategies; problem resolution.

1 Docente UNAN-Managua/FAREM-Estelí. Correo electrónico: hdonaldariel@gmail.com. (c) 2019 Revista Multi-Ensayos. 


\section{INTRODUCCIÓN}

Indagando al inicio del semestre en la web, me encontré "un método para la resolución de problemas matemáticos", al analizarlo me pareció muy fundamental e importante, principalmente porque los problemas matemáticos relacionados a la vida diaria, es una habilidad básica que los estudiantes deben de tener a lo largo de sus vidas, y que deberán usarla frecuentemente cuando salgan de la universidad y que más con la aplicación de un método que facilite este proceso.

La estadística l está estrechamente relacionada con el método científico, en la recopilación, organización, presentación y análisis de datos; tanto para obtener conclusiones como para tomar decisiones con base en los análisis efectuados. La importancia de la estadística radica en su aplicación en todas las carreras que ofrece la Facultad de Ciencias Económicas, por lo que se pretende que el estudiante logre obtener las bases de la estadística descriptiva y las probabilidades para que en un futuro pueda realizar inferencias estadísticas. (UNAN-Managua., Junio, 2013, pág. 3).

En este sentido el término estadística se refiere a datos numéricos, tales como promedios, medianas, porcentajes y números índices que ayudan a entender una gran variedad de negocios y situaciones económicas. Sin embargo, como se verá, el campo de la estadística es mucho más que datos numéricos. En un sentido amplio, la estadística se define como el arte y la ciencia de reunir datos, analizarlos, presentarlos e interpretarlos.

Especialmente en los negocios, y en la economía, la información obtenida al reunir datos, analizarlos, presentarlos e interpretarlos proporciona a directivos, administradores y personas que deben tomar decisiones una mejor comprensión del negocio o entorno económico, permitiéndoles así tomar mejores decisiones con base en mejor información. (Anderson, Sweeney, \& Williams, 2008, pág. 3).

\section{DESARROLLO}

En este escrito abordaré como se desarrollaron cada una de las unidades de la asignatura, desglosadas en dos horas clase por encuentro a la semana, para un total de dieciséis sesiones. Las unidades desarrolladas corresponden a: Unidad I: Estadística Descriptiva; Unidad II: Probabilidades; Unidad III: Distribuciones de probabilidad; y Unidad IV: Distribución Normal.

La asignatura se desarrolló aplicando una metodología participativa, basada en el aprender-haciendo. Para ello, se aplicaron técnicas, tales como: lluvias de ideas, explicación participativa, aprendizaje basado en preguntas dirigidas, ejercicios de significación o de análisis, aprendizaje colaborativo (trabajo en equipo), exposiciones, debates, análisis de situaciones del entorno y principalmente el aprendizaje basado en la resolución de problemas y por tareas, siendo estas dos últimas, constantes en todo el proceso, tal como lo sugiere el programa de Estadística I. 
Lo antes mencionado, permite un proceso pedagógico activo que integra la teoría con la práctica. Durante el desarrollo de la asignatura, fue importante el protagonismo de los estudiantes, el diálogo de saberes, intercambio de experiencias y la producción colectiva de aprendizajes en la interacción entre estudiantes y docente.

"Se cometen muchos menos errores usando datos inadecuados que cuando no se utilizan datos." Charles Babbage (1792-1871)

En la primera unidad "Estadística Descriptiva", la metodología utilizada para el desarrollo de la temática fue la siguiente:

Lluvia de ideas, permitiendo a los estudiantes dar sus aportes a través de preguntas que permiten reflexionar, motivar la curiosidad y dar ideas, acerca de un problema y de sus soluciones o, en general, sobre un tema que requiere de ideas originales; explicación participativa dando oportunidad a la realización de preguntas y comentarios sobre el tema, y sin faltar la resolución de ejercicios de significación o de análisis de forma individual y grupal.

Además, en esta unidad se realizaron exposiciones por parte de los estudiantes, lo que permitió que indagarán no solo en internet, en la biblioteca, sino también en instituciones y fábricas, sobre datos estadísticos de temáticas a exponer.

En donde, las exposiciones se realizaron al final de la unidad, y dicho trabajo investigativo se orientó al inicio del curso, para su realización de forma paralela a los temas de la primera unidad. Todo esto consistió, en que todas las temáticas abordas en el aula de clase relacionadas a la "Estadística Descriptiva", los estudiantes, investigarían un tema de su interés en el entorno social, vinculado al perfil de la carrera, y aplicar estos aprendizajes de manera práctica, yendo desde la recolección de datos hasta su respectiva interpretación.

Este trabajo investigativo permitió que los estudiantes vincularan la teoría con la práctica, ya que los temas analizados en el aula, se aplicaron de manera experimental y objetiva, permitiendo que los educandos percibieran la importancia de la estadística en su carrera, como futuros profesionales.

Al final de la unidad, los estudiantes expusieron sus investigaciones, compartiendo sus experiencias y dificultades en el proceso en forma de plenario a sus compañeros y docente, sin dejar al lado la entrega del trabajo impreso, para su respectiva evaluación.

De igual manera, para las explicaciones se hizo uso de, tablas, mapas conceptuales, gráficos, muy útiles para captar y sintetizar ideas relevantes, facilitando una rápida visualización de los contenidos de aprendizaje. Algo que como docente me llama mucho la atención de los mapas conceptuales y gráficos por que favorecen recordar lo plasmado en ellos y poder aprender de una manera organizada. 
"La vida es una escuela sobre probabilidad." Walter Bagehot (1826-1877)

En la segunda unidad "Probabilidades", para el abordaje de la temática, se desarrolló la siguiente metodología:

Recapitulación del tema anterior, lo que se realiza siempre al inicio de la clase, como una forma de llevar la secuencia y refrescar lo acontecido en la clase anterior. El docente haciendo uso de la técnica expositiva, con materiales del medio (ruleta, dados, canicas y monedas), indagó sobre los conocimientos previos de los estudiantes.

Donde se platearon preguntas relacionadas a: ¿qué es probabilidad?, ¿cuál es la importancia de la aplicación de probabilidades en la administración de empresas?, ¿qué es un evento probabilístico?, ¿qué es un espacio muestral?, entre otras, las cuales fueron analizadas de manera experimental, con materiales del medio, para así unificar ideas y aclarar dudas, respecto a conceptos básicos de la probabilidad.

Además, en esta unidad se aplicaron estrategias como, el análisis de situaciones del entorno, aprendizaje colaborativo (trabajo en equipo), aprendizaje basado en la resolución de problemas y por tareas, siendo esta última una constante, ya que el estudiantado debe poner en práctica el autoestudio.

Lo planteado anteriormente, da como resultado un importante interés por parte de los estudiantes ante la posibilidad de darle solución a problemas relacionados al perfil de la carrera o situaciones en las cuales pudiesen enfrentarse en un futuro, dentro de las empresas y la necesidad de saber con exactitud por que las probabilidades son fundamentales e importante en el campo administrativo. La facilitación de material de apoyo por medio de folletos y guías de aprendizaje, hizo posible una asimilación mejor de los contenidos.

Sin embargo, se notó que los estudiantes presentan dificultades en el análisis, ante la resolución de problemas, haciéndose la interrogante, ¿qué regla o teorema de la probabilidad aplicamos aquí?

Tal como, se menciona en la Revista EDUCACION (Ballestero, 2008, pág. 124) "Una de las áreas de la matemática que mayor dificultad adquiere para los estudiantes es la resolución de problemas; ellos son capaces de resolver mecánicamente las operaciones fundamentales básicas, pero no saben cómo aplicarlas para la solución de un problema, ya que sólo se les ha enseñado a actuar de forma repetitiva y tradicional".

A través de la resolución de los mismos en la pizarra de manera conjunta, se fue mejorando esta problemática, no en la totalidad por supuesto, ya que esto requiere de un proceso largo de disciplina, constancia y perseverancia.

La vida merece la pena sólo por dos cosas: por descubrir las matemáticas y por enseñarlas." Simeón Poisson (1781-1840). 
En la tercera unidad "Distribuciones de probabilidad", se inició con la técnica aprendizaje basado en preguntas dirigidas hacia los estudiantes con el fin de indagar sobre los conocimientos previos y relacionarlos con los nuevos, lo que conllevó a visualizar y comprender la vinculación de dicha unidad, con las anteriores.

Las distribuciones de probabilidad están relacionadas con las distribuciones de frecuencias (estadística descriptiva). De hecho, podemos pensar que una distribución probabilidad, describe la forma en que se espera varíen los resultados. Como estas distribuciones representan expectativas de que algo suceda, resultan modelos útiles para hacer inferencias y tomar decisiones en condiciones de incertidumbre. (Balderas Lozada, Valle Sotelo, \& Gómez Castillo, 2004, pág. 178).

Para ello, se llevó a cabo la estrategia del trabajo colaborativo (trabajo en equipo); donde se elaboró una serie de preguntas, guía de análisis del entorno o problemas, que permitió a los estudiantes compartir sus experiencias e ideas.

Ahora bien, mediante el trabajo colaborativo se ha podido observar que no necesariamente los que dominan más han logrado buenos resultados, sino también aquellos que lograron organizarse, aprovechando sus fortalezas y oportunidades. De igual manera, han tenido la confianza de hacer consultas sobre lo que no entienden, manteniéndose una buena relación estudiante - docente.

Sin embargo, hay que recalcar que la falta de análisis, hacia la resolución de problemas por los estudiantes, es una dificultad que se siguió evidenciando en las tareas orientadas a realizar en casa y que se comentó al inicio del ensayo. Pese a que los conceptos estadísticos son sencillos, los educandos muestran dificultades en su correcta interpretación. Este aprendizaje incompleto tiende a reflejarse en el rendimiento académico de los estudiantes, es ahí donde entró la motivación del docente, hacia la mejora de esta problemática, tanto para el presente como futuro.

“Cómo nos atrevemos a hablar de leyes del azar? ¿No es el azar la antítesis de toda ley?" Bertrand Russell (1872-1970)

Para la cuarta unidad "Distribución Normal”; (también conocida como distribución de Gauss), distribución más utilizada en la estadística.

Esta unidad ayuda a explicar y dominar la metodología para el cálculo de probabilidad de variables aleatorias continuas, y que son sumamente importantes, puesto que ocurren con gran frecuencia en las aplicaciones reales y porque desempeñan un papel fundamental en los métodos de estadística inferencial y medidas de interés para los administradores, tanto en el sector público como en el privado.

Para darle salida a los contenidos, concluir con el último acumulado y sabiendo de que el tiempo era limitado, el docente a través de la explicación participativa, se centró en la interpretación de ejercicios y proponer a los estudiantes a; investigar problemas relacionados a la administración donde se aplicará 
la distribución normal estándar, entregar en limpio y culminar con la participación en la pizarra por integrantes de cada equipo de trabajo, en el próximo encuentro.

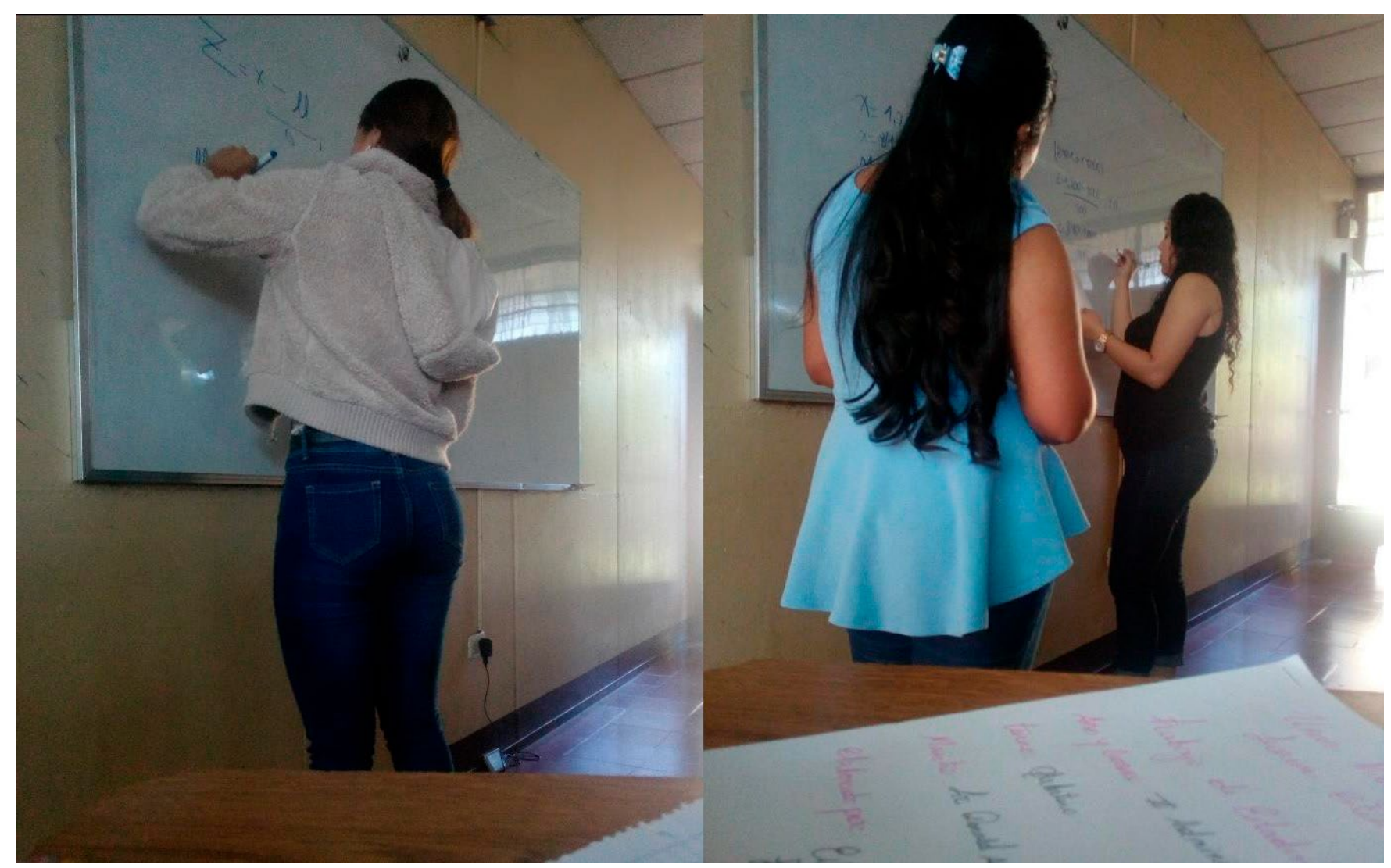

Fotos 1 y 2. Participación de estudiantes a la pizarra

Cabe mencionar, que como docente realicé un video, sobre la metodología de resolución de problemas de esta unidad, y que fue enviado al grupo de WhatsApp, a inicios de semana, con el fin de facilitar a los estudiantes la comprensión de dicho tema, lo cual se culminó con éxito en el aula de clase, con la unificación de ideas y aclaración de dudas, en forma de debate.

Del mismo modo, que la revista (NúMEROS, 2013, pág. 96), mi principal recomendación a los estudiantes es que el dominio de conocimientos que proporciona la estadística es una condición para el desempeño profesional y, por tanto, lo que no aprendan en las aulas se lo exigirá la vida en un futuro inmediato.

Para finalizar señalo el "método para la resolución de problemas matemáticos", propuesto por (Bransford \& Barry S, 1984), basado en Polya con la intención de facilitar la identificación y el reconocimiento de las distintas partes a tener en cuenta en la resolución de problemas. Las letras de la palabra IDEAL indican los elementos del método.

Las fases del método son las siguientes:

1. Identificación de los problemas: esta fase tiene la intención de ayudar a identificar los problemas.

2. Definición y representación del problema: consiste en definir y representar el problema con toda la 
precisión y cuidado que sea posible.

3. Exploración de posibles estrategias: se dirige a la indagación de distintos métodos de resolución del problema, además de analizar cómo se está reaccionando en ese momento ante el problema.

4. Actuación: fundada en una estrategia.

5. Logros: Observación y evaluación de los efectos de nuestras actividades

\section{CONCLUSIÓN}

Una de las debilidades presentadas en las exposiciones realizadas por los estudiantes, es la inseguridad al hablar (nervios) y poco dominio de algunos términos estadísticos, lo que hace que la exposición sea monótona y poco llamativa para el resto de los escuchas. Esto me lleva a reflexionar, la importancia de realizar más seguido esta técnica, para que los educandos desarrollen mejor estas habilidades.

Por ende, como docente que empiezo a ejercer esta maravillosa profesión, debo tener presente que la matemática no se aprende por transmisión directa de lo que explica el maestro o de la información que se obtiene de los libros de texto o internet; sino que se aprende en interacción con situaciones problemáticas las cuales obligan al estudiante a modificar su estructura cognitiva por el contacto con una multiplicidad de acciones que requieren distintas habilidades.

Ahora bien, las técnicas utilizadas a lo largo de la facilitación de la asignatura Estadística l, permitió desarrollar las temáticas a abordar en cada unidad. De igual manera, propició que los estudiantes interactuaran entre ellos e iniciaran el desarrollo de un análisis crítico - reflexivo, que se continuará consolidándose a lo largo de la carrera, a través del proceso de enseñanza-aprendizaje.

\section{REFERENCIAS}

Anderson, D., Sweeney, D., \& Williams , T. (2008). ESTADÍSTICA PARA ADMINISTRACIÓN Y ECONOMÍA. (10a.ed ed.). México: Cengage Learning.

Balderas Lozada, M., Valle Sotelo , J. C., \& Gómez Castillo, R. (2004). ESTADÍSTICA PARA ADMINISTRACIÓN Y ECONOMÍA (Séptima Edición ed.). Mexico: Pearson Educación.

Gorgas García , J., Cardiel López, N., \& Zamorano Calvo, J. (2011). ESTADÍSTICA BÁSICA PARA ESTUDIANTES DE CIENCIAS. Madrid, España.

Ballestero, M. M. (2008). ENSEÑANZA EFICAZ DE LA RESOLUCIÓN DE PROBLEMAS EN MATEMÁTICAS. Revista Educación, 138. San José, Costa Rica: Revista Educación 32(1), 123-138.

Bransford, J., \& Barry S, S. (1984). Obtenido de http://elviejoclub.blogspot.com/2014/12/metodo-idealde-resolucion-de- problemas.html

NúMEROS, R. d. (2013). La estadística descriptiva como herramienta de análisis en la investigación. (S. C. Matemáticas., Ed.) Vol. 84, 175. Obtenido de http://www.sinewton.org/numeros

UNAN-Managua., U. n. (Junio, 2013). Programa de Asignatura Estadística I. Managua, Nicaragua. 